\title{
Um caso de hipertiroidismo na grávida: suplementação excessiva de iodo?
}

Joana Ramos Nóbrega, ${ }^{1}$ Ana Cebolais ${ }^{2}$

\section{RESUMO}

A suplementação de iodo (150 a $200 \mu \mathrm{g} / \mathrm{dia})$ foi recomendada às mulheres em fase pré-concecional, grávidas e em amamentação exclusiva. Após introdução deste suplemento aumentou a incidência de autoimunidade tiroideia, hipotiroidismo e de hipertiroidismo, por tireotoxicose induzida por iodo em pessoas com bócio multinodular pré-existente.

O artigo descreve uma situação de hipertiroidismo na grávida, procurando ilustrar dificuldades diagnósticas e alertar para a importância de pensar no problema, principalmente em contexto das atuais recomendações de suplementação de iodo na gravidez. $O$ caso descrito refere-se a uma mulher de 38 anos com antecedentes familiares de bócio multinodular, com gravidez gemelar, que fez suplementação com iodo através de dois suplementos (o prescrito + um multivitamínico), perfazendo uma dose muito acima do recomendado. Conhecer as várias fontes de iodo que a grávida ingere e os fatores de risco para desenvolvimento de patologia tiroideia torna-se fundamental.

Existe controvérsia sobre fazer ou não estudo a todas as grávidas ou em mulheres com alto risco de disfunção da tiroide. A triagem universal pré-concecional não é recomendada, mas alguns peritos são da opinião que se deve dosear a TSH em situações de risco. A gestação múltipla e a iatrogenia estão entre as causas de hipertiroidismo na gravidez. Idade superior a 30 anos, antecedentes pessoais de bócio ou familiares de disfunção tiroideia constituem recomendaçães para estudo de disfunção tiroideia nas mulheres em fase pré-concecional e grávidas.

Nesta grávida não foi realizado estudo pré-concecional da função tiroideia nem no primeiro trimestre da gravidez. A suplementação com iodo foi realizada excedendo as recomendações. Este artigo salienta a necessidade em conhecer os fatores de risco antes da prescrição de iodo na gravidez e pré-conceção, assim como realça o risco de sobredosagem através da toma de múltiplos suplementos, alguns de venda livre em farmácias ou para-farmácias.

Palavras-chave: Iodo; Gravidez; Tiroide; Disfunção tiroideia.

\section{INTRODUÇÃO}

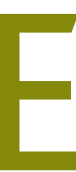

m 2013, a Direção-Geral da Saúde (DGS) publicou a Orientação Técnica n. ${ }^{\circ} 011 / 2013$, recomendando a suplementação de iodo sob a forma de iodeto de potássio (150 a $200 \mathrm{~g} /$ dia) às mulheres em fase pré-concecional, grávidas e em amamentação exclusiva. ${ }^{1}$ Tal orientação baseia-se no facto de o iodo ser essencial para a síntese de hormonas tiroideias, necessárias ao adequado desenvolvimento do sistema nervoso central durante a vida fetal e consequente normal neuro-desenvolvimento da criança. ${ }^{1}$

O feto está dependente do aporte materno de iodo até

1. Médica Interna de Medicina Geral e Familiar. USF Conchas. 2. Médica Assistente de Medicina Geral e Familiar. USF Conchas. às 20 semanas de gestação, sendo por isso importante a suplementação desde a fase de pré-conceção. ${ }^{1-3} \mathrm{Na}$ grávida, a necessidade de iodeto diário cresce $50 \%$, especialmente no primeiro trimestre., ${ }^{2,4}$

Em 2010 e 2012 foram publicados os resultados do maior estudo transversal com análise da iodúria nas grávidas portuguesas, envolvendo um total de $3.631 \mathrm{mu}-$ lheres vigiadas nos cuidados de saúde secundários. Estes resultados poderão não ser representativos da população geral, uma vez que representam utentes de maior risco clínico; no entanto, revelaram níveis inferiores aos considerados satisfatórios pela Organização

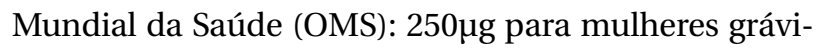
das ou a amamentar. ${ }^{2}$ Somente $17 \%$ das grávidas de Portugal Continental apresentavam níveis de iodúria satis- 
fatórios e as mulheres residentes no interior do país tinham um défice mais acentuado do que as do litoral. Entre as grávidas dos arquipélagos existia um défice ainda mais evidente: $92 \%$ das grávidas na Madeira apresentavam baixa iodúria, subindo para $99 \%$ nos Açores. Globalmente, a média de iodúria situou-se em $82,5 \mu \mathrm{g} / \mathrm{dL}$, valor que corresponde a uma região de défice ligeiro. ${ }^{5-6}$

No Minho foram realizados dois estudos cujos resultados demonstraram que mesmo as mulheres em idade fértil apresentavam valores deficitários de iodúria. Também os níveis de hormonas tiroideias entre a maioria das grávidas eram insuficientes, atendendo aos padrões estabelecidos pela OMS.7-8

O impacto das alterações da função tiroideia em idade fértil e na gravidez é inegável. Cerca de 6 a $20 \%$ das mulheres em idade reprodutiva apresentam anticorpos antitiroideus e têm risco aumentado de infertilidade inexplicada, de abortos espontâneos, nascimentos prematuros e disfunção tiroideia no pós-parto. ${ }^{4,9-11} \mathrm{O}$ hipotiroidismo na gravidez, com uma prevalência de 2$3 \%$, pode ser responsável por risco aumentado de aborto, pré-eclâmpsia, restrição do crescimento intrauterino e parto pré-termo. O hipertiroidismo tem menor prevalência $(0,1-0,4 \%)$ e, enquanto o hipertiroidismo clínico acarreta risco aumentado de aborto, pré-eclâmpsia, restrição do crescimento intrauterino e parto pré-termo (Quadro I), o subclínico parece não estar associado a outcomes negativos da gravidez. ${ }^{4,9-11}$

As alterações fisiológicas durante a gravidez podem mimetizar alguns dos sinais observados no hipertiroidismo, incluindo o aumento no metabolismo basal, frequência cardíaca, fadiga, ansiedade, palpitações, sopro cardíaco, intolerância ao calor, tremores nas mãos e, por conseguinte, o diagnóstico de hipertiroidismo durante a gravidez pode provocar dificuldades aos clínicos. ${ }^{9-11}$

A breve descrição de uma situação de hipertiroidismo, subclínico, numa grávida, procura alertar para a importância de refletir no problema do hipertiroidismo na gravidez e suas causas, principalmente no contexto das atuais recomendações de suplementação de iodo na gravidez.

\section{DESCRIÇÃO DO CASO}

RL, 38 anos, antecedentes obstétricos de um parto de termo e de um aborto espontâneo no $1^{\circ}$ trimestre, sem outros antecedentes pessoais relevantes, pertencente a família nuclear na fase II do ciclo de vida de Duvall. An-

\begin{tabular}{|c|c|}
\hline $\begin{array}{l}\text { QUADRO I. Consequências do } \\
\text { gravidez (adaptado) }{ }^{11}\end{array}$ & pertiroidismo na \\
\hline Maternas & Fetais \\
\hline Aborto & \multirow{3}{*}{$\begin{array}{l}\text { Baixo peso ao nascer: } \\
\text { (prematuridade, } \\
\text { restrição intrauterina) }\end{array}$} \\
\hline Parto pré-termo & \\
\hline Insuficiência cardíaca congestiva & \\
\hline Tempestade tiroideia & Bócio \\
\hline \multirow[t]{2}{*}{ Descolamento da placenta } & Hipotiroidismo \\
\hline & Hipertiroidismo \\
\hline
\end{tabular}

tecedentes familiares maternos de bócio multinodular pós tiroidite.

Vem à primeira consulta de saúde materna na USF com a idade gestacional de sete semanas e cinco dias, portadora de ecografia que confirmava gravidez evolutiva gemelar, tendo iniciado seguimento em consulta de obstetrícia privada. Referia queixa de cansaço, mas ao exame objetivo apresentava-se corada e hidratada, apirética, normotensa, com pulso regular e rítmico e com estabilidade ponderal em relação à última pesagem. Foi encaminhada para seguimento na consulta materno-fetal do Hospital de Santa Maria (HSM).

Manteve também seguimento com a sua médica de família, permanecendo constante a queixa de cansaço. Este sintoma foi interpretado pela médica como decorrente das alterações da gravidez, agravadas por um contexto profissional e familiar exigente. No entanto, o cansaço associado ao desenvolvimento de insónia intermédia e ao aparecimento de cefaleias tipo tensão motivaram a emissão de certificado de incapacidade para o trabalho, com consequente melhoria da sintomatologia com o repouso.

Às 16 semanas de gestação vem à consulta preocupada por alterações da função tiroideia. Tinha mantido seguimento em consulta de obstetrícia privada e, segundo a utente, num contexto de tosse persistente foram-lhe pedidas análises que revelaram TSH $<0,01 \mu \mathrm{UI} / \mathrm{mL}$.

$\mathrm{Na}$ altura esclareceu que além dos suplementos de iodo e ácido fólico prescritos, se encontrava também a tomar um suplemento multivitamínico contendo 150ug de iodo. No HSM passou a ser seguida também na consulta de endocrinologia (analiticamente TSH 0,01, FT4 0,97, anticorpos antitiroideus antitiroglobu- 
QUADRO II. Causas de hipertiroidismo na gravidez (adaptado) $^{11}$

\section{Causas de hipertiroidismo na gravidez}

\begin{tabular}{l|l}
\hline Doença de Graves & Hiperemese gravídica \\
\hline Adenoma tóxico & Gestação múltipla \\
\hline Tiroidite subaguda & Mola hidatiforme \\
\hline latrogenia & \\
\hline
\end{tabular}

lina 294, anticorpos antitiroideus anti-TPO 151). Foram suspensos os suplementos contendo iodo e realizada vigilância da função tiroideia, que normalizou, sem necessidade de intervenção. Como intercorrência, saliente-se o diagnóstico de diabetes gestacional no segundo trimestre, controlada com dieta alimentar e insulinoterapia a partir das 30 semanas.

$\mathrm{O}$ parto ocorreu às 37 semanas e cinco dias, por cesariana, com nascimento de gémeos saudáveis e sem malformações, com rastreio de doenças metabólicas sem alterações. Os gémeos completaram dois anos e têm apresentado um desenvolvimento estaturo-ponderal e psicomotor adequados.

Um mês e meio após o parto, RL já tinha valores normais na prova de tolerância à glicose e mantinha função tiroideia normalizada. Atualmente, a utente mantém-se eutiroideia (TSH 1,37) e com valores de glicemia normais. Ecografia tiroideia revelou duas formações nodulares à esquerda com 4,1mm e 3,2mm.

\section{COMENTÁRIO}

Após o início da suplementação com iodo verificou-se um aumento da incidência de hipertiroidismo como resultado do desenvolvimento de tireotoxicose induzida por iodo em pessoas com bócio multinodular pré-existente, mas também em indivíduos sem patologia nodular conhecida. ${ }^{12-13}$ Um outro efeito colateral potencial da suplementação de iodo é o agravamento ou indução de tiroidite autoimune, sendo que a remoção da fonte de iodo em excesso é normalmente associada à restauração da função tiroideia. ${ }^{12-13}$

No caso descrito, a grávida estava a fazer suplementação de iodo mas através de dois suplementos: um com 262 microgramas de iodeto de potássio; e um multivitamínico com 150 microgramas. Assim sendo, esta-

\begin{tabular}{l} 
QUADRO III. Recomendações para rastreio de disfunção \\
tiroideia nas mulheres em fase pré-concecional e \\
grávidas (adaptado) ${ }^{14}$ \\
$>30$ anos \\
\hline História familiar de disfunção tiroideia \\
\hline Bócio \\
\hline Anticorpos tiroideus positivos, principalmente antiperoxidase \\
\hline Sintomas ou sinais de hipotiroidismo \\
\hline Diabetes mellitus tipo 1 ou outras doenças autoimunes \\
\hline Infertilidade ou reprodução assistida \\
\hline Antecedentes de parto pré-termo \\
Antecedentes de irradiação pescoço ou cabeça ou cirurgia \\
tiroideia \\
\hline Terapêutica para disfunção tiroideia
\end{tabular}

va a fazer um total de $412 \mathrm{~g} /$ dia de iodeto de potássio, o que corresponde a um valor muito acima do recomendado (150 a $200 \mathrm{~g} /$ dia) pela OMS e DGS.

No Quadro II estão representadas as principais causas de hipertiroidismo na gravidez, onde se incluem a iatrogenia e a gravidez gemelar. Neste caso clínico, a gravidez gemelar pode ser a justificação para o desenvolvimento de hipertiroidismo. No entanto, esta grávida encontrava-se a fazer suplementação com iodo em dosagem acima da recomendada e desenvolveu hipertiroidismo subclínico, tendo normalizado valores de TSH após interrupção dos suplementos. Salienta-se, assim, a importância de conhecer as várias fontes de iodo que a grávida está a fazer.

A propósito deste caso clínico surgiu a dúvida: existirá indicação para pesquisar doença tiroideia em todas as grávidas e mulheres em fase pré-concecional? Ou devem-se apenas realizar testes de deteção de casos em mulheres com alto risco de disfunção da tiroide?

O tema é controverso, mas segundo diretrizes da American Thyroid Association não existe evidência suficiente para recomendação a favor ou contra a triagem universal através da TSH no primeiro trimestre de gravidez, mas as mulheres com elevado risco de doença tiroideia (Quadro III) podem beneficiar da pesquisa de disfunção tiroideia. ${ }^{15}$ A The Endocrine Society, em 2012, publicou orientações no sentido de não ser recomendada, antes da gravidez, uma triagem universal de 
mulheres saudáveis para a disfunção da tiroide, mas afirma que os clínicos devem identificar mulheres com alto risco (Quadro III) e efetuar medição de TSH. ${ }^{14} \mathrm{Em}$ relação à gravidez não houve consenso e alguns membros recomendam a triagem de todas as mulheres grávidas pela 9a semana ou no momento da sua primeira visita, enquanto outros recomendam deteção de casos de alto risco (Quadro III). ${ }^{14}$

O rastreio universal para anticorpos antitiroideus antes ou durante a gravidez não está recomendado. Contudo, existe uma associação positiva entre a presença de anticorpos e o aumento do aborto, parto pré-termo ou progressão para doença tiroideia. Portanto, se estes forem identificados, as mulheres devem ser rastreadas para eventuais alterações da TSH antes da gravidez, bem como durante o primeiro e segundo trimestres da gravidez. ${ }^{14}$

Nesta grávida não foi realizado estudo da função tiroideia pré-concecional, apesar dos seus antecedentes familiares e idade, que constituíam fatores de risco para disfunção tiroideia. Também não foi solicitada TSH nas análises do primeiro trimestre, apesar da gravidez gemelar e da queixa de cansaço, interpretado como sintoma inespecífico relacionado com o contexto da utente e não relacionado com eventual desenvolvimento de disfunção tiroideia.

A suplementação com iodo foi realizada e a dose tomada pela grávida excedeu as recomendações. Será que a realização precoce do estudo da função tiroideia podia ter identificado algum risco ou problema? Será que o hipertiroidismo subclínico se desenvolveu devido a iatrogenia?

Este artigo não dará certamente respostas exatas, mas procura alertar para a importância de conhecer os fatores de risco antes da prescrição de iodo na gravidez e pré-conceção, assim como para a necessidade de uma rigorosa avaliação da medicação habitual ao longo de toda a gravidez, a fim de evitar os riscos associados à toma de múltiplos suplementos vitamínicos, alguns dos quais de venda livre em farmácias ou para-farmácias.

\section{REFERÊNCIAS BIBLIOGRÁFICAS}

1. Direção-Geral da Saúde. Aporte de iodo em mulheres na preconceção, gravidez e amamentação: orientação n. ${ }^{\circ}$ 011/2013, de 26/08/2013. Lisboa: DGS; 2013

2. World Health Organization. Assessment of iodine deficiency disorders and monitoring their elimination: a guide for programme managers. 3rd ed. Geneva:WHO; 2007. ISBN 9789241595827
3. Yarrington C, Pearce EN. lodine and pregnancy. JThyroid Res. 2011;2011: 934104.

4. Nazarpour S, Ramezani Tehrani F, Simbar M, Azizi F. Thyroid dysfunction and pregnancy outcomes. Iran J Reprod Med. 2015;13(7):387-96.

5. Limbert E, Prazeres S, São Pedro M, Madureira D, Miranda A, Ribeiro M, et al. lodine intake in Portuguese pregnant women: results of a countrywide study. Eur J Endocrinol. 2010;163(4):631-5.

6. Limbert E, Prazeres S, Madureira D, Miranda A, Ribeiro M, Abreu FS, et al.Aporte do iodo nas Regiões Autónomas da Madeira e dos Açores [lodine intake in the Autonomous Regions of Madeira and Azores]. Rev Port Endocrinol Diabetes Metab. 2012;7(2):2-7. Portuguese

7. Costeira MJ, Oliveira P, Ares S, de Escobar GM, Palha JA. lodine status of pregnant women and their progeny in the Minho Region of Portugal. Thyroid. 2009;19(2):157-63.

8. Costeira MJ, Oliveira P, Ares S, Roque S, de Escobar GM, Palha JA. Parameters of thyroid function throughout and after pregnancy in an iodine-deficient population. Thyroid. 2010;20(9):995-1001.

9. Cignini P, Cafà EV, Giorlandino C, Capriglione S, Spata A, Dugo N. Thyroid physiology and common diseases in pregnancy: review of literature. J Prenat Med. 2012;6(4):64-71.

10. Van den Boogaard E, Vissenberg R, Land JA, van Wely M, van der Post JA, Goddijn M, et al. Significance of (sub)clinical thyroid dysfunction and thyroid autoimmunity before conception and in early pregnancy: a systematic review. Hum Reprod Update. 2011;17(5):605-19.

11. Mestman JH. Hyperthyroidism in pregnancy. Best Pract Res Clin Endocrinol Metab. 2004;18(2):267-88.

12. Fiore $E$, Tonacchera $M$, Vitti P. Influence of iodization programmes on the epidemiology of nodular goitre. Best Pract Res Clin Endocrinol Metab. 2014;28(4):577-88.

13. Aakre I, Bjøro T, Norheim I, Strand TA, Barikmo I, Henjum S. Development of thyroid dysfunction among women with excessive iodine intake: a 3-year follow-up. J Trace Elem Med Biol. 2015;31:61-6.

14. De Groot L, Abalovich M, Alexander EK, Amino N, Barbour L, Cobin RH, et al. Management of thyroid dysfunction during pregnancy and postpartum: an Endocrine Society clinical practice guideline. J Clin Endocrinol Metab. 2012;97(8):2543-65.

15. Stagnaro-Green A, Abalovich M, Alexander E, Azizi F, Mestman J, Negro $R$, et al. Guidelines of the American Thyroid Association for the diagnosis and management of thyroid disease during pregnancy and postpartum. Thyroid. 2011;21(10):1081-125.

16. Spencer L, Bubner T, Bain E, Middleton P. Screening and subsequent management for thyroid dysfunction pre-pregnancy and during pregnancy for improving maternal and infant health. Cochrane Database Syst Rev. 2015;(9):CD011263.

\section{CONFLITO DE INTERESSES}

Os autores declaram não ter quaisquer conflitos de interesse.

\section{ENDEREÇO PARA CORRESPONDÊNCIA}

Joana Ramos Nóbrega

E-mail: joana.m.nobrega@gmail.com

http://orcid.org/0000-0003-1671-2451

Recebido em 17-02-2017

Aceite para publicação em 09-02-2019 


\section{ABSTRACT}

\section{A CASE OF HYPERTHYROIDISM IN PREGNANCY: EXCESSIVE IODINE SUPPLEMENTATION?}

In Portugal, iodine supplementation is recommended for all women in pre-conception, during pregnancy and exclusive breastfeeding in the dose of 150 to $200 \mu \mathrm{g} /$ day. After this recommendation, we found an increase of the incidence of autoimmune thyroiditis, both hypo and of hyperthyroidism, by iodine-induced thyrotoxicosis in people with pre-existing multinodular goiter.

This article reports a case of hyperthyroidism in a pregnant woman, aiming to describe the difficulty on diagnosis and to alert to the importance of take this situation in mind, especially in the context of current recommendations for iodine supplementation in pregnancy.

The case reports a twin pregnancy in a 38-year-old woman with family history of multinodular goiter. She was supplemented with iodine through two supplements: the one prescribed by the doctor and another one in a multivitamin pill over-thecounter. The total dose was much higher than recommended, leading to a iatrogenic hyperthyroidism.

There's no consensus about screening of thyroid dysfunction before supplementation, although some experts defend to ask for preconceptional thyroid-stimulating hormone (TSH), especially in woman at risk, like age over 30 years, personal or family history of goiter or other thyroid disease.

Multiple gestation and iatrogenic events are the leading causes of hyperthyroidism in pregnancy.

It's crucial to know the various sources of iodine and the total amount ingested by pregnants, especially if there is risk for thyroid disease. Many pregnants are taking supplements over-the-counter, with the false-perception of security, because it's not a medication.

In this case, there was no previous record of TSH in pre-conception or during pregnancy and total iodine supplementation was excessive, justifying the events, and calling for increased attention for both factors.

Keywords: lodine; Pregnancy; Thyroid; Thyroid dysfunction. 